Périurbanisation différentielle : mutations et reorganisation de l'espace à l'est de la région dakaroise (Diamniadio, Sangalkam et Yene), Sénégal

\title{
Momar Diongue
}

\section{OpenEdition}

Journals

Édition électronique

URL : http://journals.openedition.org/cdg/2085

DOI : $10.4000 / \mathrm{cdg} .2085$

ISSN : 2107-7266

\section{Éditeur}

UMR 245 - CESSMA

Référence électronique

Momar Diongue, «Périurbanisation différentielle : mutations et reorganisation de l'espace à l'est de la région dakaroise (Diamniadio, Sangalkam et Yene), Sénégal », Carnets de géographes [En ligne], 1 | 2010, mis en ligne le 01 octobre 2010, consulté le 24 septembre 2020. URL : http:// journals.openedition.org/cdg/2085; DOI : https://doi.org/10.4000/cdg.2085

Ce document a été généré automatiquement le 24 septembre 2020.

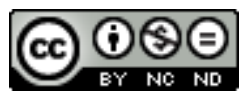

La revue Carnets de géographes est mise à disposition selon les termes de la Licence Creative Commons Attribution - Pas d'Utilisation Commerciale - Pas de Modification 4.0 International. 


\title{
Périurbanisation différentielle : mutations et reorganisation de l'espace à l'est de la région dakaroise (Diamniadio, Sangalkam et Yene), Sénégal
}

\author{
Momar Diongue
}

Dans un contexte de réarticulation sélective du local au global, la ville ouest-africaine en général et sénégalaise en particulier enregistre des mutations accélérées qui affectent les dynamiques spatiales et sociales, les processus de production, les cadres comme les types de gestion. La presqu'île du Cap-Vert est engagée dans un processus de métropolisation qui dépasse l'échelle de l'agglomération dakaroise stricto sensu. L'agglomération elle-même est marquée par deux processus : spatial par une densification horizontale et verticale, et économique par sur une redistribution des activités et des différentes catégories sociales dans la ville par les mécanismes du marché. Á l'échelle de la presqu'île du Cap-Vert, l'État fait preuve d'un nouveau volontarisme en affichant plus d'ambitions dans la production de l'espace urbain. Dans la capitale, ces ambitions se déclinent principalement sous forme de grands projets d'infrastructures et de développement économique et parfois symboliques. D'abord consacrée à l'amélioration des axes Nord-Sud, la dynamique des grands projets a été ensuite orientée vers l'axe Ouest-Est. En terme spatial, l'avenir de la métropole dakaroise se joue au niveau de l'axe Ouest-Est en raison de la configuration géographique. Les mauvaises liaisons entre l'Ouest et l'Est de la presqu'île en termes de réseaux d'infrastructures, surtout routières et d'assainissement, pèsent aujourd'hui de manière négative sur l'efficacité du système urbain dakarois.

2 Par ces grands projets, l'État veut reconfigurer le système urbain dakarois, et accroître son efficacité. Cette approche étatique de la question urbaine par "grands projets " change la nature des relations entre l'agglomération dakaroise et ses périphéries où 
sont localisées la plupart des opérations prévues. Cet enjeu métropolitain positionne l'espace périurbain au centre du problème. Les choix de localisation comme la dimension foncière des grands projets ont des effets sur l'évolution et la gestion du périurbain dakarois. Ces dynamiques à l'œuvre dans la presqu'île du Cap-Vert rendent très actuels les questionnements sur la périurbanisation. La périurbanisation de l'Est de la presqu'île est-elle est train de rompre avec la dynamique de banlieurisation résidentielle jusqu'ici dominante ? Cette redistribution des fonctions urbaines préfigure-t-elle une métropolisation polycentrique ? Est-ce le temps du déploiement de nouvelles fonctions productives urbaines au profit d'un rééquilibrage en faveur des périphéries métropolitaines, avec une intégration fonctionnelle de l'ensemble et non un éclatement ? Ce renouveau d'intérêt étatique pour la ville sénégalaise est-elle en phase avec le processus de décentralisation et le transfert de la gestion urbaine à l'échelle locale. Le retour étatique est-il le signe d'un pouvoir central plus fort, doté d'une politique urbaine plus ambitieuse, de plus de moyens et de capacités pour la mettre en œuvre en épaulant les collectivités locales dans la gestion des espaces urbains et périurbains ? Traduit-il simplement le signe éphémère du renouveau d'un pouvoir politique prenant l'allure d'un contre-pied au processus de décentralisation en reléguant les pouvoirs publics locaux au second plan ? L'intervention étatique dans ce contexte pose la question des relations non seulement entre les intérêts propres de l'État et ceux des pouvoirs publics locaux, mais aussi entre ces derniers. La question de la gouvernance des espaces urbains et périurbains est donc posée.

\section{Une approche territoriale comparative de la périurbanisation}

3 D'où notre choix en faveur d'une approche territoriale qui associe périurbanisation et décentralisation. Notre question centrale est l'émergence de territoires dans le périurbain, les logiques qui sous-tendent leurs dynamiques et leurs effets sur la gouvernance territoriale et le développement local. L'intérêt de cette approche consiste à ne plus prendre la périurbanisation comme une finalité de la recherche, mais comme le point de départ d'une analyse devant conduire à une réflexion d'ordre théorique. Une telle approche cadre avec une démarche comparative axée sur les processus de périurbanisation et de décentralisation à l'Est de la région dakaroise.

\section{Démarche méthodologique : des territoires aux espaces témoins, des espaces témoins aux localités, des localités aux unités spatiales}

4 La comparaison de deux processus dans plusieurs lieux, espaces et territoires en situation périurbaine, permet de concilier une analyse pratique et théorique de l'objet d'étude en s'accrochant le plus possible à la réalité du terrain. Nous partons des activités non agricoles de l'espace - le développement résidentiel et des activités économiques - pour étudier l'évolution du périurbain dakarois, ses mécanismes, les logiques et les pratiques des acteurs à l'origine de celle-ci.

5 Nous avons privilégié trois échelles de lecture dans l'analyse. Á l'échelle micro (des unités non agricoles et des localités), il s'agit d'appréhender les dynamiques de 
déploiement spatial de la périurbanisation en fonction des contextes locaux. Á l'échelle des collectivités locales et du périurbain, le développement économique et résidentiel est mis en relation à des logiques d'acteurs et des pratiques gestionnaires pour questionner les mécanismes d'évolution de l'espace et leurs effets sur la gestion et le développement des territoires. Comment la périurbanisation participe à l'enclenchement, à la consolidation, à l'affaiblissement et/ou à la dynamisation des processus de territorialisation? L'objectif est d'analyser les capacités de régulation de la périurbanisation et de ses effets. Enfin, à l'échelle macro, les observations et les analyses sont replacées dans le processus de métropolisation dakaroise pour s'interroger sur les enjeux, à l'échelle de la presqu'île, de l'aménagement et de la gouvernance. Les méthodes statistiques et qualitatives ont été mobilisées dans l'étude de la périurbanisation dans trois espaces témoins.

\section{Diamniadio, Sangalkam et Yene : trois espaces témoins propice à l'observation, l'analyse et la comparaison des processus}

Diamniadio, Sangalkam et Yene, trois espaces témoins situés à l'Est de la région dakaroise, constituent le terrain d'observation des processus. Des positionnements géoéconomiques différenciés caractérisent Diamniadio (fonction circulatoire), Sangalkam (zone économique agricole) et Yene (sociale). Des contextes périurbains différents, qui posent la question du poids des déterminants locaux dans la périurbanisation. Que peuvent nous apporter ces trois contextes locaux dans la compréhension des processus de périurbanisation et de territorialisation?

Fiche informative

Discipline

Géographie

Directeur

Alain Dubresson

Université

Paris Ouest Nanterre La Défense

Membres du jury de thèse, soutenue le 3 juin 2010

- Alain Dubresson, Professeur à l'université Paris Ouest Nanterre La Défense

(directeur de thèse)

- Jean Fabien Steck, Maître de Conférences à l'université Paris Ouest Nanterre La

Défense

- Michel Lesourd, Professeur à l'Université de Rouen (rapporteur)

- Monique Poulot, Professeur à l'université Paris Ouest Nanterre La Défense

- Sid-Ahmed Souiah, Professeur à l'université de Cergy Pontoise (rapporteur)

Situation professionnelle

Laboratoire GECKO, Paris Ouest Nanterre La Défense 


\section{Contact}

Dionguem[at]hotmail.com

INDEX

Thèmes : Carnets de soutenances 